\title{
Resonant absorption of a short-pulse laser in a doped dielectric
}

\author{
L. K. Ang, Y. Y. Lau, ${ }^{\text {a) }}$ and R. M. Gilgenbach \\ Department of Nuclear Engineering and Radiological Sciences, University of Michigan, Ann Arbor, \\ Michigan 48109-2104
}

(Received 18 December 1998; accepted for publication 17 March 1999)

\begin{abstract}
A simple model is used to calculate the energy absorption efficiency when a laser of short pulse length impinges on a dielectric slab that is doped with an impurity with a resonant line at the laser frequency. It is found that the energy absorption efficiency is maximized for a certain degree of doping concentration (at a given pulse length) and also for a certain pulselength (at a given doping concentration). Dimensionless parameters are constructed, allowing calculations with one set of parameters be used to infer the results expected for other sets of parameters. (C) 1999 American Institute of Physics. [S0003-6951(99)02720-5]
\end{abstract}

Absorption of laser light in a dielectric remains a problem of considerable practical interest. With the advent of chirped pulse lasers ${ }^{1}$ and free electron lasers, ${ }^{2}$ short laser pulses on the order of 100s of optical cycles are produced. These short laser pulses are poised to find numerous applications including spectroscopy, material processing, charged particle acceleration, inertial fusion, and even ophthalmological procedures. ${ }^{3-8}$ The free electron laser is highly tunable by the energy of the electron beam, while the chirped pulse laser offers considerable flexibility in adjusting the pulse length. In this letter, we analyze the amount of laser energy that can be absorbed in a dielectric slab. We are particularly interested in the enhanced heating when the dielectric is doped with an impurity that has a resonant line at the laser frequency.

We assume that the dielectric is lossless, with relative dielectric constant $\epsilon$ when the impurities are absent. We use the Drude model to account for the impurities of density $N$ with a corresponding plasma frequency $\omega_{p}$. Numerically, $\omega_{p}=2 \pi \times 9 \mathrm{THz} \times\left[\mathrm{N} / 10^{18} \mathrm{~cm}^{-3}\right]^{1 / 2}$. The impurity is assumed to have a resonant frequency $\omega_{0}$ and a finite linewidth $\omega_{0} / Q$, where we have used the quality factor $Q$ whose inverse represents the width of the resonance. In this model, the refractive index, $n$, then takes on the usual form,

$$
n^{2}=\epsilon-\frac{\omega_{p}^{2}}{\omega^{2}-j \omega \omega_{0} / Q-\omega_{0}^{2}},
$$

where $\omega$ is the laser frequency. To calculate the amount of laser energy that is absorbed in a dielectric slab of width $L$ with the refractive index given by Eq. (1), we first establish the reflection coefficient $(R)$ and the transmission coefficient $(T)$, both in amplitude, for a plane wave of single frequency $\omega$ (with $e^{j \omega t}$ dependence) incident normally onto the dielectric. These coefficients depend on $n$ and $\omega L$ and are easily deduced:

$$
R=\frac{1}{D}\left(n^{2}-1\right)(B-1 / B),
$$

\footnotetext{
${ }^{a)}$ Electronic mail: yylau@umich.edu
}

$$
T=\frac{4 n}{D},
$$

where

$$
\begin{aligned}
& B=e^{-j n \omega L / c}, \\
& D=-(1-n)^{2} B+(1+n)^{2} / B,
\end{aligned}
$$

and $c$ is the speed of light in vacuum.

Assume that the energy content of the incident laser pulse is unity. The absorption coefficient $A$ (in energy) may be determined from energy conservation. It is equal to the incident wave energy, which is unity, minus the reflected wave energy and the transmitted wave energy. For a laser pulse with a Gaussian shape in time, $A$ becomes

$$
A=\frac{\tau}{\sqrt{2 \pi}} \int_{0}^{\infty} d \omega e^{-\left(\omega-\omega_{E}\right)^{2} \tau^{2} / 2}\left[1-|R(\omega)|^{2}-|T(\omega)|^{2}\right],
$$

where $\omega_{E}$ is the center frequency and $\tau$ is the pulse length of the laser. While Eq. (6) is intuitively obvious, it may be established using the Parseval's theorem, which converts the energy content representation in time domain to frequency domain. Without loss of generality, we have considered only the positive frequency components $(\omega>0)$ in Eq. (6), hence Eq. (1) may be approximated by

$$
n^{2} \cong \epsilon-\frac{\omega_{p}^{2}}{2 \omega_{0}\left(\omega-\omega_{0}-j \omega_{0} / 2 Q\right)} .
$$

In taking the square root of Eq. (7) to obtain the refractive index $n=n_{r}+j n_{i}$, the proper branch needs to be taken (the real part $n_{r}>0$ and the imaginary part $n_{i}<0$ ).

Before we present the numerical results, we note the following regarding the single-frequency absorption coefficient $\mathcal{A}=1-|R|^{2}-|T|^{2}$. It can be shown from Eqs. (2)-(5) that $\mathcal{A}=0$ if the refractive index $n$ is either purely real or purely imaginary. It then follows from Eq. (1) or Eq. (7) that $\mathcal{A}=0$ if $\omega_{p}=0$ or if $Q$ is infinite. This means that resonant absorption by the impurities is due to their finite linewidth at the transition frequency. The amount of resonant absorption then depends on the overlap between the laser spectrum and the resonance spectrum of the impurities. 


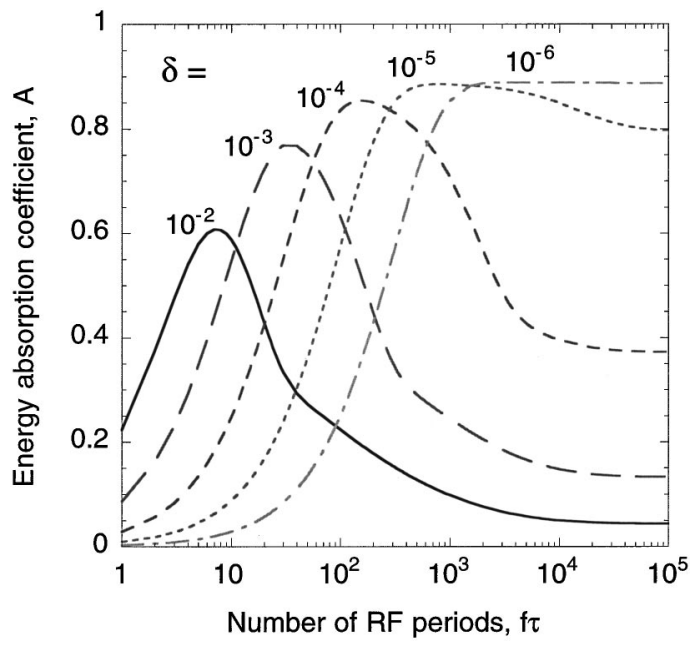

FIG. 1. Energy absorption coefficient $(A)$ as a function of the pulse length $(f \tau)$ expressed in units of optical periods, at various values of normalized impurity concentration $(\delta)$. The values of $\delta=10^{-6}, 10^{-5}, \ldots, 10^{-2}$, correspond, respectively, to impurity density $N=1.43 \times 10^{17}, \quad 1.43$ $\times 10^{18}, \ldots, 1.43 \times 10^{21} \mathrm{~cm}^{-3}$. Here, we set $L / \lambda=1000$, i.e., $L=0.248 \mathrm{~mm}$.

We next identify the dimensionless parameters in the calculation of $A$, so that calculation for one set of parameters may be used to infer the results for another set of parameters. The integration over the frequency $\omega$ in Eq. (6), with a change of variable, may be converted to an integration over the dimensionless dummy variable $y$, where $y=(\omega$ $\left.-\omega_{0}\right) /\left(\omega_{0} \delta\right)$. The dimensionless parameter $\delta$ measures the impurity concentration and is defined, along with other dimensionless parameters, as

$$
\delta=\frac{\omega_{p}^{2}}{2 \epsilon \omega_{0}^{2}}, \quad \Lambda=\omega_{0} \tau \delta / \sqrt{2}, \quad \rho=\frac{\omega_{E}-\omega_{0}}{\omega_{0} \delta}, \quad \kappa=\frac{L}{\lambda \sqrt{\epsilon}}
$$

where $\lambda=2 \pi c / \omega_{0}$ is the free space wavelength. In Eq. (8), $\Lambda$ measures the laser pulse length, $\rho$ measures the separation

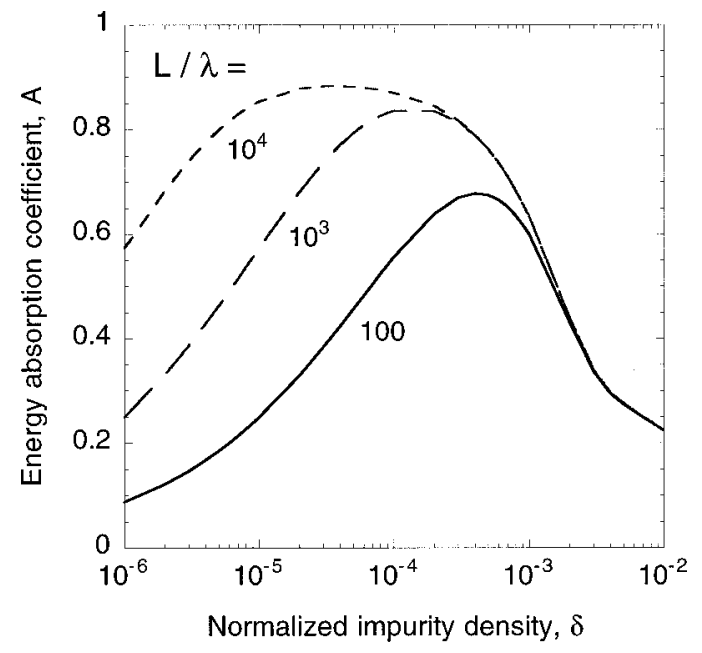

FIG. 2. Energy absorption coefficient $(A)$ as a function of the normalized impurity concentration $(\delta)$, at various slab width $(L / \lambda)$ expressed in units of free space wavelength. The values of $\delta=10^{-6}, 10^{-5}, \ldots, 10^{-2}$, correspond, respectively, to impurity density $N=1.43 \times 10^{17}, 1.43 \times 10^{18}, \ldots, 1.43$ $\times 10^{21} \mathrm{~cm}^{-3}$. Here, we set $f \tau=100$.

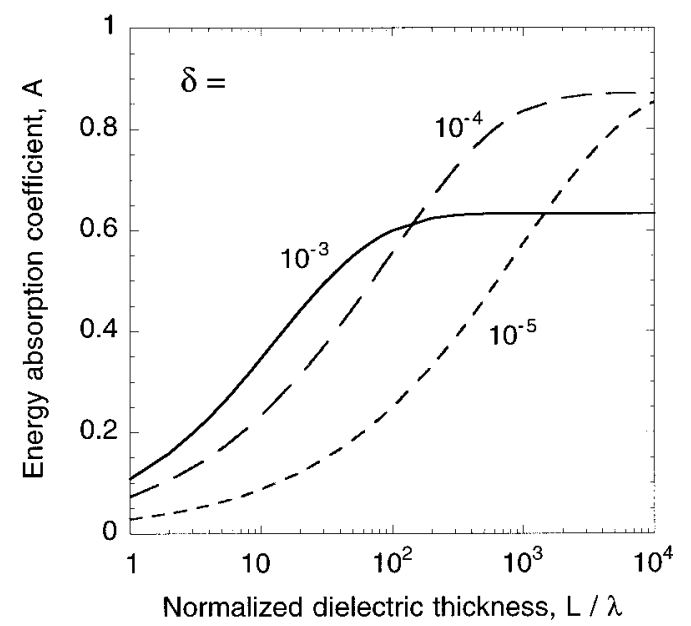

FIG. 3. Energy absorption coefficient $(A)$ as a function of the normalized slab width $(L / \lambda)$ expressed in units of free space wavelength, at various normalized impurity concentrations $(\delta)$. The values of $\delta=10^{-5}, 10^{-4}$, $10^{-3}$, correspond, respectively, to impurity density $N=1.43 \times 10^{18}, 1.43$ $\times 10^{19}, 1.43 \times 10^{20} \mathrm{~cm}^{-3}$. Here, we set $f \tau=100$.

between the laser frequency and the dielectric dopant transition frequency, and $\kappa$ measures the width of the dielectric slab.

In terms of the dimensionless quantities, Eq. (7) gives

$$
n=\sqrt{\epsilon} \sqrt{1-\frac{1}{y-j /(2 Q \delta)}},
$$

while Eq. (6) may be shown to yield the energy absorption coefficient $A$ with the following dependence on the dimensionless parameters:

$$
A=A(\Lambda, Q \delta, \kappa, \epsilon, \rho) .
$$

In Eq. (10), the least important parameter is $\epsilon$, which we set equal to 4 henceforth. For simplicity, we set $\rho=0$. That is, we assume that the laser frequency coincides with the transition frequency. We then examine the dependence on the remaining three parameters. It is interesting to note that the resonant linewidth and the concentration of the impurity enter in the product $(Q \delta)$ in Eqs. (9) and (10). As we have

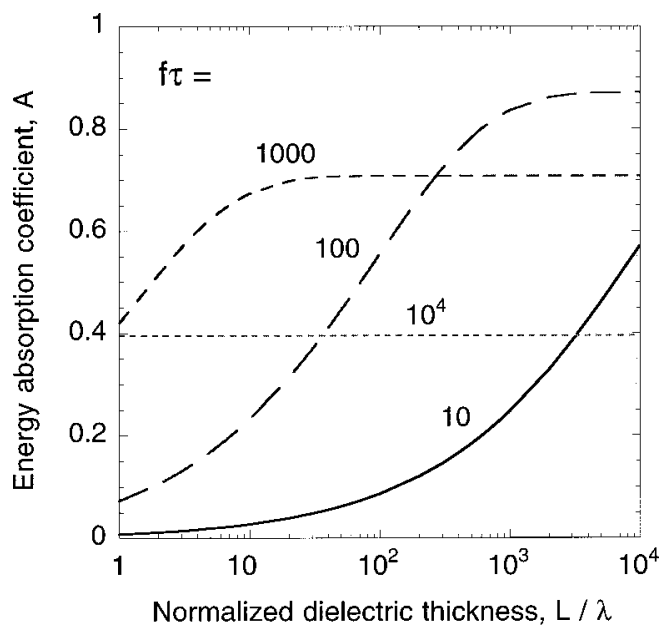

FIG. 4. Energy absorption coefficient $(A)$ as a function of the normalized slab width $(L / \lambda)$ expressed in units of free space wavelength, at various normalized pulse length $(f \tau)$. Here we set $\delta=10^{-4}$, i.e., impurity density $N=1.43 \times 10^{19} \mathrm{~cm}^{-3}$. 
remarked above, there can hardly be any absorption if $Q$ becomes infinity or $\delta$ becomes zero. Thus, A increases from zero, reaches some maximum value, and decreases back to zero as the product $Q \delta$ increases from zero to infinity. The last statement implies that there is an optimal doping to yield a maximum energy absorption efficiency for a given pulse length. Moreover, since the amount of absorption depends on the degree of overlap between the spectra in the resonance line and in the laser pulse, it is then not surprising that there is an optimal pulse length for maximum efficiency in the energy absorption.

To show the dependence of the energy absorption coefficient on the pulse length, doping concentration, and the width of the dielectric, we will consider a specific example with $\epsilon=4, \omega_{E}=\omega_{0}=2 \pi c /(248 \mathrm{~nm})$, which corresponds to a $\mathrm{KrF}$ laser and a resonance line at $248 \mathrm{~nm}$. We arbitrarily assign a value of $Q=248 \mathrm{~nm} / 0.005 \mathrm{~nm}=5 \times 10^{4}$, corresponding to a resonant linewidth of $0.005 \mathrm{~nm}$. While these parameters are chosen rather arbitrarily, the numerical results obtained for them may be used to predict other cases with different sets of parameters through the scaled dependence exhibited in Eq. (10). The scaled dependence in the combination of $Q \delta$, for example, enables us to assess the effects of the resonant linewidth even if the precise value or the nature of the linewidth remain uncertain.

Shown in Fig. 1 is the energy absorption coefficient $(A)$ versus the laser pulse length, measured in units of optical cycle, for various doping density $N$. For a moderately high doping density, there is an optimal pulse length for maximum energy efficiency of absorption. As the pulse length tends to infinity, the absorption coefficient $A$ approaches $\mathcal{A}$, the single-frequency absorption coefficient. This is expected because a longer pulse is closer to being monochromatic. Note also from Fig. 1 that a higher doping concentration does not necessarily imply a higher energy absorption efficiency. This is clearly illustrated in Fig. 2 which shows that $A$ is maximized at some doping density for a pulse length of 100 optical cycles. This optimal density depends on the width of the dielectric slab. This dependence on the slab width is shown in Fig. 3 for various doping densities, and in Fig. 4 for various pulse lengths. It is interesting to note from Figs. 1-4 that tens of percent of the laser energy can be resonantly absorbed with a modest amount of impurities.
The applicability of the simple model of resonant absorption may be tested with a tunable laser whose frequency can be adjusted to coincide with the resonance line of the impurities embedded in the dielectric. Indeed, by varying the doping concentration or the laser pulse length, one might even infer the intrinsic linewidth from the measured absorption coefficients. On a much more modest scale, we found experimentally ${ }^{9}$ that, when a copper vapor laser (510 and $578 \mathrm{~nm})$ shines on a transparent dielectric (polymethylmethacrylate-Lucite), the light becomes strongly absorbed if the dielectric surface is painted with ink (propynol based solvent Color Index blue 70). One may well wonder whether such greatly enhanced absorption is of the resonant type studied in this letter. Because of scaled parameters, our model may be applicable to gas-phase absorption by short pulse lasers or microwaves; an example of the latter source would be a $100 \mathrm{~ns}$ microwave pulse at $1.3 \mathrm{GHz} .{ }^{10} \mathrm{It}$ could also be applicable to the semiconductor industry, as it may be used to determine the proper amount of photosensitive chemicals in photoresist, when various lithography wavelengths (e.g., 436, 365, 248, $193 \mathrm{~nm}$, etc.) are used.

The authors wish to thank Professor Mary Brake for useful discussions on resonance linewidths. This work was supported by NSF, DOE, and AFOSR/MURI.

${ }^{1}$ M. D. Perry and G. Mourou, Science 264, 917 (1994).

${ }^{2}$ J. M. J. Madey, H. A. Schwettman, and W. M. Fairbank, IEEE Trans. Nucl. Sci. 20, 980 (1973).

${ }^{3}$ C. W. Roberson and P. Sprangle, Phys. Fluids B 1, 3 (1989); E. Esarey, P. Sprangle, J. Krall, and A. Ting, IEEE Trans. Plasma Sci. PS-24, 252 (1996).

${ }^{4}$ M. Tabak, J. Hammer, M. E. Glinsky, W. L. Kruer, S. C. Wilks, J. Woodworth, E. M. Campbell, M. D. Perry, and R. J. Mason, Phys. Plasmas 1, 1626 (1994).

${ }^{5}$ X. Liu, D. Du, and G. Mourou, IEEE J. Quantum Electron. 33, 1706 (1997).

${ }^{6}$ M. J. Kelley, Nucl. Instrum. Methods Phys. Res. B 144, 186 (1998).

${ }^{7}$ W. Kautek, S. Mitterer, J. Kruger, W. Husinsky, and G. Grabner, Appl. Phys. A: Solids Surf. 58, 513 (1994).

${ }^{8}$ R. Srinivasan and B. Braren, Appl. Phys. A: Solids Surf. 45, 289 (1988).

${ }^{9}$ P. L. G. Ventzek, R. M. Gilgenbach, C. H. Ching, R. A. Lindley, and W. B. McColl, J. Appl. Phys. 72, 3080 (1992).

${ }^{10}$ K. J. Hendricks, P. D. Coleman, R. W. Lemke, M. J. Arman, and L. Bowers, Phys. Rev. Lett. 76, 154 (1996). 